University of Nebraska - Lincoln

DigitalCommons@University of Nebraska - Lincoln

\title{
First-principles studies of a two-dimensional electron gas at the interface in ferroelectric oxide heterostructures
}

\author{
Yong Wang \\ University of Nebraska - Lincoln \\ Manish K. Niranjan \\ University of Nebraska-Lincoln, manish@iith.ac.in \\ Sitaram Jaswal \\ University of Nebraska, sjaswal1@unl.edu \\ Evgeny Y. Tsymbal \\ University of Nebraska-Lincoln, tsymbal@unl.edu
}

Follow this and additional works at: https://digitalcommons.unl.edu/physicstsymbal

Part of the Condensed Matter Physics Commons

Wang, Yong; Niranjan, Manish K.; Jaswal, Sitaram; and Tsymbal, Evgeny Y., "First-principles studies of a two-dimensional electron gas at the interface in ferroelectric oxide heterostructures" (2009). Evgeny Tsymbal Publications. 27.

https://digitalcommons.unl.edu/physicstsymbal/27

This Article is brought to you for free and open access by the Research Papers in Physics and Astronomy at DigitalCommons@University of Nebraska - Lincoln. It has been accepted for inclusion in Evgeny Tsymbal Publications by an authorized administrator of DigitalCommons@University of Nebraska - Lincoln. 


\title{
First-principles studies of a two-dimensional electron gas at the interface in ferroelectric oxide heterostructures
}

\author{
Yong Wang, Manish K. Niranjan, S. S. Jaswal, and Evgeny Y. Tsymbal \\ Department of Physics and Astronomy, Nebraska Center for Materials and Nanoscience, \\ University of Nebraska, Lincoln, Nebraska 68588, USA \\ (Received 9 July 2009; published 29 October 2009)
}

\begin{abstract}
The discovery of a two-dimensional electron gas (2DEG) at the interface between two insulating oxides has recently stimulated intense research activity in this field. The 2DEG has unique properties that are promising for applications in all-oxide electronic devices. For such applications it is desirable to have the ability to control 2DEG properties by external stimulus. Here we employ first-principles calculations to investigate $\mathrm{KNbO}_{3} / A \mathrm{TiO}_{3}(001)(A=\mathrm{Sr}, \mathrm{Pb}$, and $\mathrm{Ba})$ heterostructures where perovskites ferroelectrics, $\mathrm{KNbO}_{3}, \mathrm{PbTiO}_{3}$, and $\mathrm{BaTiO}_{3}$, are used as oxide constituents to create the interface 2DEG. Our results suggest that the polar $\left(\mathrm{NbO}_{2}\right)^{+} /(A \mathrm{O})^{0}$ interface in these heretostructures favors the formation of 2DEG similar to that at the $(\mathrm{LaO})^{+} /\left(\mathrm{TiO}_{2}\right)^{0}$ interface in a $\mathrm{LaAlO}_{3} / \mathrm{SrTiO}_{3}$ heterostructure. We predict that the presence of spontaneous ferroelectric polarization which can be switched between two stable states allows modulations of the carrier density and consequently the conductivity of the 2DEG. The effect occurs due to the screening charge at the interface that counteracts the depolarizing electric field and depends on polarization orientation. The magnitude of the effect of polarization on the 2DEG properties strongly depends on contrast between polarizations of the two constituents of the heterostructure: the larger is the difference in the two polarizations, the bigger is the effect. For a sufficiently large polarization difference, we predict a metal-insulator transition at the interface driven by polarization reversal. This behavior is found for the $\mathrm{KNbO}_{3} / \mathrm{PbTiO}_{3}$ interface and for the $\mathrm{KNbO}_{3} / \mathrm{BaTiO}_{3}$ interface when polarizations of $\mathrm{KNbO}_{3}$ and $\mathrm{BaTiO}_{3}$ are antiparallel. The proposed concept of ferroelectrically controlled interface conductivity may be very interesting for memory and logic applications and we hope that our predictions will stimulate experimental studies in this field.
\end{abstract}

DOI: 10.1103/PhysRevB.80.165130

\section{INTRODUCTION}

In recent years, oxide heterostructures with atomically abrupt interfaces have been experimentally realized due to the advance in the thin-film-deposition techniques such as laser deposition and molecular-beam epitaxy. Physical properties and a rich spectrum of physical phenomena may arise at the interfaces of these heterostructures that are not found in either of their bulk counterparts. ${ }^{1,2}$ A prominent example is the recent discovery of the formation of a metallic phase at the interface between two oxide insulators, $\mathrm{SrTiO}_{3}$ and $\mathrm{LaAlO}_{3}{ }^{3}$ It was found that this metallic phase is confined within a couple of nanometers near the interface ${ }^{4}$ and therefore can be regarded as a two-dimensional electron gas (2DEG). The 2DEG has a very high carrier density and a relatively high carrier mobility making it attractive for applications in nanoelectronics, e.g., as all-oxide field-effect transistors. ${ }^{5,6}$ Furthermore, in such interfacial 2DEGs other functionalities involving magnetic ${ }^{7}$ and superconducting ${ }^{8}$ phenomena at low temperature have also been reported. These interesting properties of the 2DEG at oxide interfaces have stimulated significant research activity both in experiment ${ }^{9-15}$ and in theory. ${ }^{16-27}$

There are two main scenarios to explain the metallic state observed at the $\mathrm{LaO} / \mathrm{TiO}_{2}$ interface of $\mathrm{SrTiO}_{3} / \mathrm{LaAlO}_{3}$ heterostucture: electronic reconstruction ${ }^{28}$ and oxygen vacancies. ${ }^{10-12} \mathrm{LaAlO}_{3}$ is formed of alternately charged atomic layers $(\mathrm{LaO})^{+}$and $\left(\mathrm{AlO}_{2}\right)^{-}$and $\mathrm{SrTiO}_{3}$ is composed of neutral layers $(\mathrm{SrO})^{0}$ and $\left(\mathrm{TiO}_{2}\right)^{0}$. The electronic potential diverges with $\mathrm{LaAlO}_{3}$ thickness due to the intrinsic polar planes in $\mathrm{LaAlO}_{3}$ and polar discontinuity at interface
PACS number(s): 73.20.-r, 71.30.+h, 77.80.Fm, 77.84.Dy

$(\mathrm{LaO})^{+} /\left(\mathrm{TiO}_{2}\right)^{0}$. In semiconductor heterointerfaces such a polar discontinuity is well known and causes the interface atomic reconstruction to avoid the diverging electrostatic potential. $^{29,30}$ But transition metals in perovskites can have mixed valence making electronic reconstruction possible, which is more favorable in energy than atomic reconstruction and eliminates the divergence of potential. ${ }^{26}$ Half an electron per two-dimensional unit cell is transferred through the interface from $\mathrm{LaAlO}_{3}$ to $\mathrm{SrTiO}_{3}$ causing the interfacial titanium ion in $\mathrm{SrTiO}_{3}$ to be in the mixed-valence state $\left(\mathrm{Ti}^{+3.5}\right)$. This half an electron at the $(\mathrm{LaO})^{+} /\left(\mathrm{TiO}_{2}\right)^{-0.5}$ interface forms the 2DEG. Oxygen vacancies also dope $\mathrm{SrTiO}_{3}$ substrate with electrons and make the interface conducting. ${ }^{10-12}$ However, at high oxygen pressure, oxygen vacancies may be avoided and the dominant reason of conducting interface in this situation is the electronic reconstruction. ${ }^{26}$ In some systems the polar discontinuity may be eliminated by the competition between the electronic- and atomic-reconstruction mechanisms. ${ }^{31,32}$

Analogous to the ionic-polar discontinuity, the 2DEG may be created at an interface due to electric-polarization discontinuity. This has been observed in $\mathrm{ZnO} / \mathrm{Mg}_{\mathrm{x}} \mathrm{Zn}_{1-\mathrm{x}} \mathrm{O}$ heterostructures where a sheet charge is formed at the interface to relax the electrostatically unfavorable state. ${ }^{33,34}$ An interesting possibility is to combine the two discontinuities at the interface by using a ferroelectric oxide material as one (or both) constituents of the dig heterostructure. Due to reversible polarization of the ferroelectric, the dig properties of such a functional interface may be controlled by ferroelectric polarization switching. ${ }^{35}$ This physical phenomenon expands recently predicted effects of ferroelectric polarization on 


\begin{tabular}{|c|}
\hline$\left(\mathrm{NbO}_{2}\right)^{+}$ \\
\hline$(\mathrm{KO})^{-}$ \\
\hline$\left(\mathrm{NbO}_{2}\right)^{+}$ \\
\hline$(\mathrm{KO})^{-}$ \\
\hline$\left(\mathrm{NbO}_{2}\right)^{+}$ \\
\hline$(\mathrm{AO})^{0}$ \\
\hline$\left(\mathrm{TiO}_{2}\right)^{0}$ \\
\hline$(\mathrm{AO})^{0}$ \\
\hline$(\mathrm{TiO})^{0}$ \\
\hline$(\mathrm{AO})^{0}$ \\
\hline
\end{tabular}

(a)

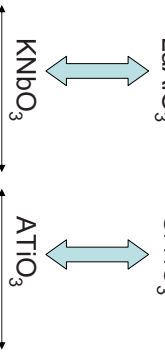

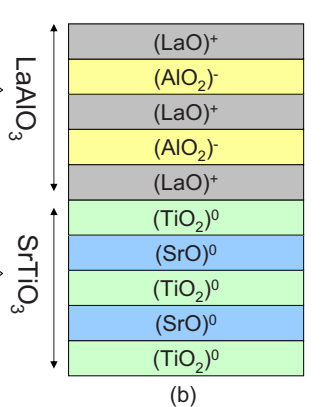

(b)
FIG. 1. (Color online) $\left(\mathrm{NbO}_{2}\right)^{+} /(A \mathrm{O})^{0}$ interface in (a) $\mathrm{KNbO}_{3} / A \mathrm{TiO}_{3}$ heterostructure as an analog of $(\mathrm{LaO})^{+} /\left(\mathrm{TiO}_{2}\right)^{0}$ interface in (b) $\mathrm{LaAlO}_{3} / \mathrm{SrTiO}_{3}$ heterostructure.

functional properties of thin-film oxide heterostructures such as the interface magnetoelectric effect, ${ }^{36-38}$ the tunneling electroresistance effect ${ }^{39-41}$ and the effect ferroelectricity on tunneling magnetoresistance. ${ }^{40,42}$

In this paper, we investigate oxide heterostructures composed of ferroelectric constituents to study the effect of ferroelectricity on the interface properties. The particular systems chosen for this study are $\mathrm{KNbO}_{3} / A \mathrm{TiO}_{3}(001)(A=\mathrm{Sr}$, $\mathrm{Pb}$, and $\mathrm{Ba}$ ) oxide heterostructures. Ferroelectric $\mathrm{KNbO}_{3}$ has alternating charge layers of $\left(\mathrm{NbO}_{2}\right)^{+}$and $(\mathrm{KO})^{-}$along the [001] direction similar to those of $\mathrm{LaAlO}_{3}$ as shown in Fig. 1. $A \mathrm{TiO}_{3}$ consists of alternating planes of $\left(\mathrm{TiO}_{2}\right)^{0}$ and $(A \mathrm{O})^{0}$ which are analogous to the neutral planes in the $\mathrm{SrTiO}_{3}$. Similar to $(\mathrm{LaO})^{+} /\left(\mathrm{TiO}_{2}\right)^{0}$, the polar discontinuity at the $\left(\mathrm{NbO}_{2}\right)^{+} /(A \mathrm{O})^{0}$ interface in the $\mathrm{KNbO}_{3} / A \mathrm{TiO}_{3}$ heterostructure leads to potential divergence which needs to be removed by electron transfer. Thus the $\left(\mathrm{NbO}_{2}\right)^{+} /(\mathrm{SrO})^{0}$ interface in $\mathrm{KNbO}_{3} / A \mathrm{TiO}_{3}$ is expected to be compensated electronically leading to the conducting interface. On the other hand, the presence of the free charge at the interfaces allows for the growth of a superlattice with stable polarized regions and large polarization discontinuities at the internal interfaces, as was shown recently using first-principles calculations for a $\mathrm{SrTiO}_{3} / \mathrm{KNbO}_{3}$ system. ${ }^{43}$

In our previous paper ${ }^{35}$ we have shown that the $2 \mathrm{DEG}$ is indeed formed at the $\left(\mathrm{NbO}_{2}\right)^{+} /(A \mathrm{O})^{0}$ interface and the conducting properties of the $2 \mathrm{DEG}$ can be switched by the orientation of the spontaneous polarization. We demonstrated that electronic properties of the 2DEG are affected by the polarization due to the formation of the screening charge at the interface, counteracting the polarization charge and hence the depolarizing electric field. The screening charge depends on polarization orientation and consequently 2DEG can be switched between two conduction states by polarization reversal. This paper expands our previous findings to include details of the electronic and atomic structures of the $\mathrm{KNbO}_{3} / A \mathrm{TiO}_{3}$ interfaces, which are important to elucidate the mechanisms responsible for the modulation of the $2 \mathrm{DEG}$ properties by ferroelectric polarization. We consider the possibility of the antiparallel polarization in the heterostructures with all ferroelectric constituents producing a stronger effect on the 2DEG formed at the interface. Finally, we discuss a possibility of the controllable metal-insulator transition at the interface driven by polarization reversal.

The paper is organized as follows. Section II describes the computation details and defines the construction of studied structures. Section III presents the electronic and atomic properties of $\mathrm{KNbO}_{3} / A \mathrm{TiO}_{3}(001)(A=\mathrm{Sr}, \mathrm{Pb}$, and $\mathrm{Ba})$ oxide heterostructures, emphasizing the polarization effect on the properties of $2 \mathrm{DEG}$. Section IV summarizes the results of this work.

\section{THEORETICAL APPROACH}

\section{A. Computational method}

Our theoretical studies are performed using densityfunctional theory (DFT) (Refs. 44 and 45) implemented within the pseudopotential plane-wave method ${ }^{46,47}$ and the Vienna $a b$ initio simulation package (VASP). ${ }^{48}$ The projected augmented wave method ${ }^{49}$ is used to approximate the electron-ion potential. The exchange-correlation effects are treated within the Perdew-Burke-Ernzerhoff ${ }^{50}$ form of the generalized gradient approximation (GGA). The electron wave functions are expanded in a plane-wave basis set limited by a cut-off energy of $500 \mathrm{eV}$. Spin-orbit corrections are not included in the calculations. The bulk and superlattice calculations are performed using the $6 \times 6 \times 6$ and $8 \times 8 \times 1$ Monkhorst-Pack $^{51} k$-point mesh, respectively. The selfconsistent calculations are converged to $10^{-5} \mathrm{eV} /$ cell and the structures are relaxed until the forces on the ions are less $0.02 \mathrm{eV} / \AA$.

\section{B. Bulk perovskites}

We consider the superlattices grown on (001) $\mathrm{SrTiO}_{3}$ single-crystal substrates. ${ }^{3}$ Thus in-plane lattice constant of the superlattice is fixed to the experimental lattice constant of $\mathrm{SrTiO}_{3}$ which is $a=3.905 \AA .{ }^{52}$ By varying the out-of-plane lattice constant $c$ in a paraelectric configuration and minimizing the total energy, the lattice constant $c$ of bulk $\mathrm{BaTiO}_{3}$, $\mathrm{SrTiO}_{3}$, and $\mathrm{KNbO}_{3}$ are calculated to be 4.143, 3.983, and $4.049 \AA$, respectively. The obtained tetragonal structures of the perovskites are used as building blocks of all the superlattices with constituents in paraelectric states with mirror plane symmetry and with spontaneous polarization where reflection symmetry is broken. ${ }^{53}$ However, the out-of-plane lattice constant $c$ of $\mathrm{PbTiO}_{3}$ without ionic relaxation $(4.018 \AA)$ is used only in superlattice in paraelectric state. With large polarization in ferroelectric state, the out-of-plane lattice constant of $\mathrm{PbTiO}_{3}$ in ferroelectric state will be much different from that in paraelectric state. When the ferroelectric state is developed in $\mathrm{PbTiO}_{3}$ in superlattice, we used out-ofplane lattice constant of $4.530 \AA$ obtained by minimizing the total energy with full ionic relaxation. Here the tetragonal distortion $c / a$ is 1.16 , which is consistent with the GGA result of Ref. 54 but overestimates the experimental value $c / a=1.06$ at room temperature. ${ }^{55}$ It is known that the GGA approximation, in general, overestimates the tetragonal distortion in ferroelectrics (see, e.g., Ref. 56), however the predicted value of polarization of $\mathrm{PbTiO}_{3}$ is consistent with experimental data as discussed in the next paragraph.

When the bulk $\mathrm{ATiO}_{3}$ structures with the in-plane constraint $a=3.905 \AA$ are in the ferroelectric state, the polarizations of $\mathrm{BaTiO}_{3}, \mathrm{KNbO}_{3}$, and $\mathrm{PbTiO}_{3}$ obtained by using the Berry-phase method ${ }^{57}$ are $0.43,0.41$, and $1.16 \mathrm{C} / \mathrm{m}^{2}$, respectively. These values are consistent with the experimental 


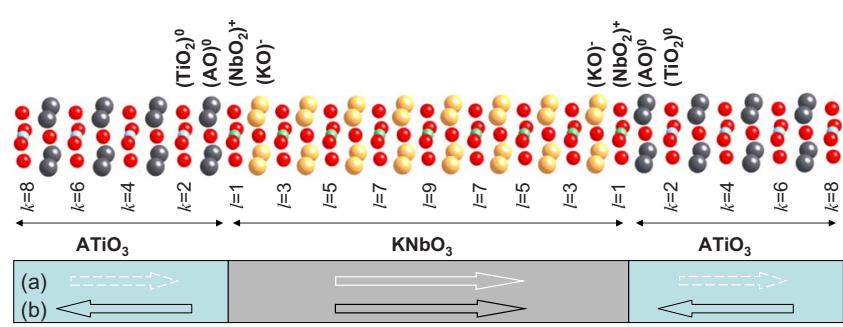

FIG. 2. (Color online) Atomic structure of the unit cell and the $\left(\mathrm{NbO}_{2}\right)^{+} /(A \mathrm{O})^{0}$ interfaces in a $\left(\mathrm{KNbO}_{3}\right)_{8.5} /\left(A \mathrm{TiO}_{3}\right)_{8.5}(001)$ superlattice with (a) parallel and (b) antiparallel polarization indicated by white and black arrows, respectively.

values of $0.46,{ }^{58} 0.37,{ }^{59}$ and $0.97 \mathrm{C} / \mathrm{m}^{2},{ }^{60}$ for polarizations of $\mathrm{BaTiO}_{3}, \mathrm{KNbO}_{3}$ and $\mathrm{PbTiO}_{3}$, respectively, and the previous theoretical calculations. ${ }^{61,53,54}$ It is notable that the calculated polarization of $\mathrm{PbTiO}_{3}$ is much larger than the calculated polarization of both $\mathrm{KNbO}_{3}$ and $\mathrm{BaTiO}_{3}$, which has an important implication for our results as discussed in Sec. III.

The calculated band gaps of bulk $\mathrm{KNbO}_{3}, \mathrm{SrTiO}_{3}$, $\mathrm{BaTiO}_{3}$, and $\mathrm{PbTiO}_{3}$ with the in-plane constraint are very close in magnitude, i.e., 2.0, 2.0, 1.8, and $2.0 \mathrm{eV}$, respectively. These values are lower than the experimental values of $3.3,{ }^{62} 3.3,{ }^{63} 3.3$, and $3.4 \mathrm{eV},{ }^{64}$ respectively, due to wellknown deficiency of DFT calculations. However, similar to our calculations they are very close in magnitude. This fact has an important implication for the formation of $2 \mathrm{DEG}$ at the $\mathrm{KNbO}_{3} / A \mathrm{TiO}_{3}(A=\mathrm{Sr}, \mathrm{Pb}$, and $\mathrm{Ba})$ interfaces, which makes it different from the $\mathrm{LaAlO}_{3} / \mathrm{SrTiO}_{3}$ interfaces, where both the calculated [3.7 eV (Ref. 25)] and the experimental [5.6 eV (Ref. 64)] gaps in $\mathrm{LaAlO}_{3}$ are much higher than those in $\mathrm{SrTiO}_{3}$.

\section{Structural model for superlattices}

We employ the periodic boundary condition to superlattice. With building blocks obtained above, the superlattices are constructed as $\left(\mathrm{KNbO}_{3}\right)_{m} /\left(A \mathrm{TiO}_{3}\right)_{n}(A=\mathrm{Sr}, \mathrm{Ba}$, and $\mathrm{Pb})$ with $m=8.5$ unit cells of $\mathrm{KNbO}_{3}$ and $n=8.5$ unit cells of $A \mathrm{TiO}_{3}$ along the [001] direction. Figure 2 shows the interface geometry of the superlattice. We assume that the two interfaces have the same termination, i.e., $\mathrm{NbO}_{2} / A \mathrm{O}$. This implies a nonstoichiometric $\mathrm{KNbO}_{3}$ which is terminated by the $\mathrm{NbO}_{2}$ monolayers on both sides. In this case an "extra" electron is introduced into the system due to an electron on the additional $\mathrm{NbO}_{2}$ monolayer. As we will see in Sec. III, this electronic charge is accommodated by partially occupying the conduction-band states near the interface, producing a 2DEG.

The interface separation distance in the $\mathrm{KNbO}_{3} / A \mathrm{TiO}_{3}$ heterostructure is determined by minimizing the total energy of a smaller superlattice $(m=2.5$ and $n=1.5)$ keeping the in-plane and out-of-plane lattice constants in $\mathrm{KNbO}_{3}$ and $A \mathrm{TiO}_{3}$ subunits unchanged. The left and right $\mathrm{NbO}_{2} / A \mathrm{O}$ interfaces (see Fig. 2) in the heterostructure are symmetric when both $\mathrm{KNbO}_{3}$ and $A \mathrm{TiO}_{3}$ are in paraelectric states. However, when ferroelectric states are developed, the interface to the right is equivalent to the interface to the left with electric polarization reversed. This allows us to study the effects of polarization reversal on the interface electronic properties by considering two interfaces in a single heterostructure.

First, we consider a reference paraelectric state in which lattice parameters of the two components in the heterostructure are kept the same as found above for the bulk structures. Only the interface separation is relaxed to minimize the total energy of the whole system. Next, we consider a ferroelectric state: we relax all the ions in the $\mathrm{KNbO}_{3} / A \mathrm{TiO}_{3}$ superlattices starting with the displacement pattern of the bulk tetragonal soft mode ${ }^{65,66}$ (with polarization pointing from left to right) and minimize the total energy with respect to atomic coordinates of all atoms in the heterostructures keeping the lattice constants unchanged. The ferroelectric states are stable with respect to the paraelectric states by energies -1.18 , -1.43 , and $-0.91 \mathrm{eV} /$ supercell for $\mathrm{KNbO}_{3} / \mathrm{BaTiO}_{3}$, $\mathrm{KNbO}_{3} / \mathrm{PaTiO}_{3}$, and $\mathrm{KNbO}_{3} / \mathrm{SrTiO}_{3}$ heterostructures, respectively.

Within the GGA approximation, the $\mathrm{SrTiO}_{3}$ in the superlattice shows polar atomic displacements. Since $\mathrm{SrTiO}_{3}$ is not a ferroelectric material in the bulk, we also investigate the interfaces of the $\mathrm{KNbO}_{3} / \mathrm{SrTiO}_{3}$ superlattice by fixing the atomic positions in the middle eight monolayers in $\mathrm{SrTiO}_{3}$. Finally, in $\mathrm{KNbO}_{3} / \mathrm{BaTiO}_{3}$ heterostructure, we consider polarizations in $\mathrm{KNbO}_{3}$ and $\mathrm{BaTiO}_{3}$ pointing opposite to each other in order to verify our explanation of the switching behavior and to introduce one more avenue to realize metal-insulator transition at the interface as discussed in Sec III.

\section{RESULTS AND DISCUSSION}

\section{A. $\mathrm{SrTiO}_{3} / \mathrm{KNbO}_{3}$}

First, we investigate properties of $\left(\mathrm{NbO}_{2}\right)^{+} /(\mathrm{SrO})^{0}$ interface in $\left(\mathrm{KNbO}_{3}\right)_{8.5} /\left(\mathrm{SrTiO}_{3}\right)_{8.5}$ heterostructure in a paraelectric state. Figures 3(a) and 3(d) show the density of states (DOS) projected onto $4 d$ orbitals of $\mathrm{Nb}$ atoms [Fig. 3(a)] and $3 d$ orbitals of Ti atoms [Fig. 3(d)], located at different $\mathrm{NbO}_{2}$ or $\mathrm{TiO}_{2}$ monolayers $l$ and $k$, respectively, away from the $\left(\mathrm{NbO}_{2}\right)^{+} /(\mathrm{SrO})^{0}$ interface. Apparently there are occupied conducting states at and below the Fermi energy, thus $n$-type metallic state is obtained at the interface. As is evident from Figs. 3(a) and 3(d), the occupation of these states is largest near the interface (i.e., for $l=1$ and $k=2$ ) and decreases with increasing distance from the interface. These occupied states are similar to those at the $(\mathrm{LaO})^{+} /\left(\mathrm{TiO}_{2}\right)^{0}$ interface in $\mathrm{LaAlO}_{3} / \mathrm{SrTiO}_{3}$ heterostructures. ${ }^{16-23}$ However, due to a smaller band gap in $\mathrm{KNbO}_{3}$ (the calculated value is $2.0 \mathrm{eV}$ ) as compared to that in $\mathrm{LaAlO}_{3}$ (the calculated value is 3.7 $\mathrm{eV}$ ), the $\mathrm{KNbO}_{3}$ conduction-band minimum in the $\mathrm{KNbO}_{3} / \mathrm{SrTiO}_{3}$ heterostructure lies much closer to the Fermi energy, i.e., about $0.4 \mathrm{eV}$, which makes it energetically favorable to bend and populate the conduction bands of $\mathrm{KNbO}_{3}$ that are mainly formed by the $\mathrm{Nb} 4 d$ orbitals [see Fig. 3(a)]. This is different from the $\mathrm{LaAlO}_{3} / \mathrm{SrTiO}_{3}$ heterostructure where the $\mathrm{LaAlO}_{3}$ conduction bands lie $2.6 \mathrm{eV}$ (Ref. 13) above the Femi level and consequently almost do not participate in the 2DEG formation.

Next, we investigate the interface of the $\left(\mathrm{KNbO}_{3}\right)_{8.5} /\left(\mathrm{SrTiO}_{3}\right)_{8.5}$ superlattice in a ferroelectric state 

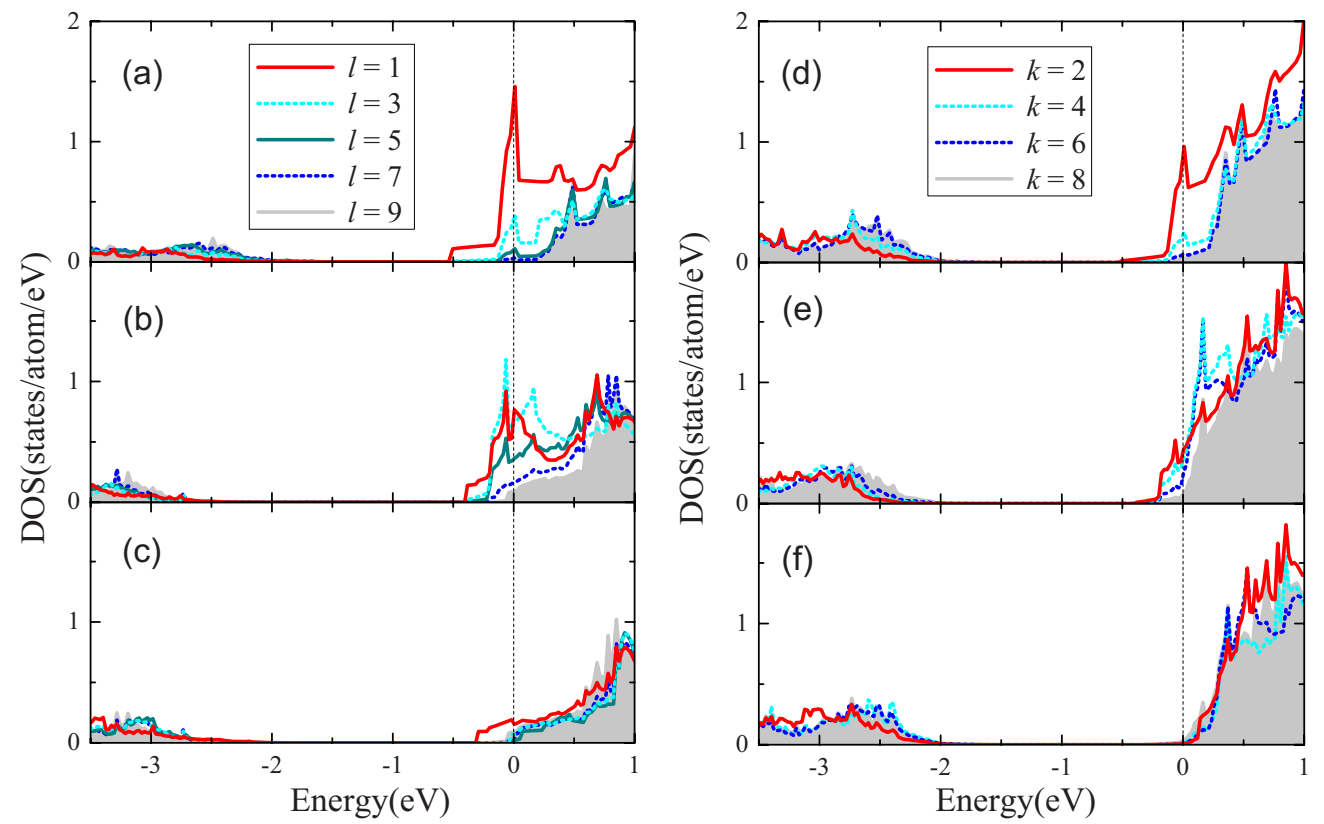

FIG. 3. (Color online) Layer-projected DOS on [(a), (b), and (c)] $4 d$ orbitals of Nb atoms and [(d), (e), and (f)] $3 d$ orbitals of Ti atoms located at different $\mathrm{NbO}_{2}$ or $\mathrm{TiO}_{2}$ monolayers $l$ or $k$ from the $\left(\mathrm{NbO}_{2}\right)^{+} /(\mathrm{SrO})^{0}$ interface, respectively, in the [(a) and (d)] $\left(\mathrm{KNbO}_{3}\right)_{8.5} /\left(\mathrm{SrTiO}_{3}\right)_{8.5}$ superlattice for paraelectric state, [(b) and (e)] ferroelectric state and right interface, and [(c) and (f)] ferroelectric state and left interface. Layer number indicates the layer counted from the interface. The shaded plots are the DOS of atoms in the central monolayer. The zero along the horizontal axis refers to the Fermi energy.

with polarization pointing from left to right. We fix eight middle monolayers of $\mathrm{SrTiO}_{3}$ and relax all the other atomic positions in the superlattice. Figure 4(a) shows the ferroelectric displacements of the cations $(\mathrm{Nb}, \mathrm{Ti}, \mathrm{K}$, and $\mathrm{Sr}$ ) relative to the oxygen anions in the heterostructure. The symmetry between the left and right interfaces [see Fig. 2(a)] is broken by the ferroelectricity resulting in the deviation between electronic structures at the two interfaces as compared to paraelectric (centrosymmetric) state. We find that ferroelectric displacements in the central monolayers of $\mathrm{KNbO}_{3}$ are close to those found in the bulk $\mathrm{KNbO}_{3}$ constrained to have the in-plane lattice constant $a=3.905 \AA$ A.

Figures 3(b), 3(c), 3(e), and 3(f) show the DOS projected onto the $\mathrm{Nb} 4 d$ orbitals and the Ti $3 d$ orbitals, respectively, located at different $\mathrm{NbO}_{2}$ or $\mathrm{TiO}_{2}$ monolayers $(l$ or $k$, respectively) from the right and left $\left(\mathrm{NbO}_{2}\right)^{+} /(\mathrm{SrO})^{0}$ interfaces. For the interface on the left [Figs. 3(b) and 3(e)], the occupancies of both $\mathrm{Nb} 4 d$ states and $\mathrm{Ti} 3 d$ states are reduced significantly as compared to the right interface [Figs. 3(c) and 3(f)] due to the ferroelectric displacements. Thus, in the superlattice the two interfaces are distinguished by the orientation of the ferroelectric polarization with respect to them. This implies that by reversing the electric polarization the carrier density of $2 \mathrm{DEG}$ at the $\mathrm{KNbO}_{3} / \mathrm{SrTiO}_{3}$ interface could be changed significantly.

For the $\mathrm{KNbO}_{3} / \mathrm{SrTiO}_{3}$ heterostructure without constrains in the middle layers of $\mathrm{SrTiO}_{3}$, we find ferroelectric-type displacements in $\mathrm{SrTiO}_{3}$ which are seen in Figs. 4(b) and 4(c). An induced polarization in $\mathrm{SrTiO}_{3}$ is known from experimental studies (see, e.g., Ref. 67). We note, however, that in our calculations these ferroelectric displacements are partially the result of using the GGA approximation which over- estimates the equilibrium lattice constant of $\mathrm{SrTiO}_{3}$, resulting in the tetragonal distortion of $\mathrm{SrTiO}_{3}$ when the experimental lattice constant is used to constrain the in-plane lattice parameter. We find, however, that for the superlattice with the unconstrained $\mathrm{SrTiO}_{3}$ the layer-resolved DOS on $\mathrm{Nb}$ and $\mathrm{Ti}$ atoms are similar to those in the superlattice where eight middle monolayers in $\mathrm{SrTiO}_{3}$ are fixed. This indicates that the induced polarization in $\mathrm{SrTiO}_{3}$ does not affect the result qualitatively due to the fact that the $\mathrm{SrTiO}_{3}$ subunit in the superlattice is a dielectric at the fringe of ferroelectricity ${ }^{4}$ with much smaller spontaneous polarization compared to $\mathrm{KNbO}_{3}$.

The switchable behavior can be understood in terms of the screening of the polarization charge achieved by changing the free-electron density of 2DEG at the interface. As discussed above the polarization in $\mathrm{SrTiO}_{3}$ can be ignored for this consideration. Polarization in $\mathrm{KNbO}_{3}$ is pointing from the left to the right causing negative and positive polarization-bound charges accumulated at left and right interfaces, respectively. To reduce the depolarization field and thus decrease the energy in the superlattice, the free charge is enhanced at the right interface and reduced at the left interface to compensate polarization charges. This is seen from Fig. 5 which shows the free charge on $\mathrm{Nb}$ and $\mathrm{Ti}$ atoms in the paraelectric (open circles) and ferroelectric (open triangles) states. These charges are obtained by integrating the layerdependent DOS shown in Fig. 3 from the conduction-band minimum up to the Fermi energy. Thus each point on the curves in Fig. 5 shows the number of free electrons on the $\mathrm{Nb}$ and $\mathrm{Ti}$ sites in the superlattice. Figure 5 also shows the screening charges (solid symbols) calculated from the difference between the free charges in the ferroelectric and paraelectric states. 

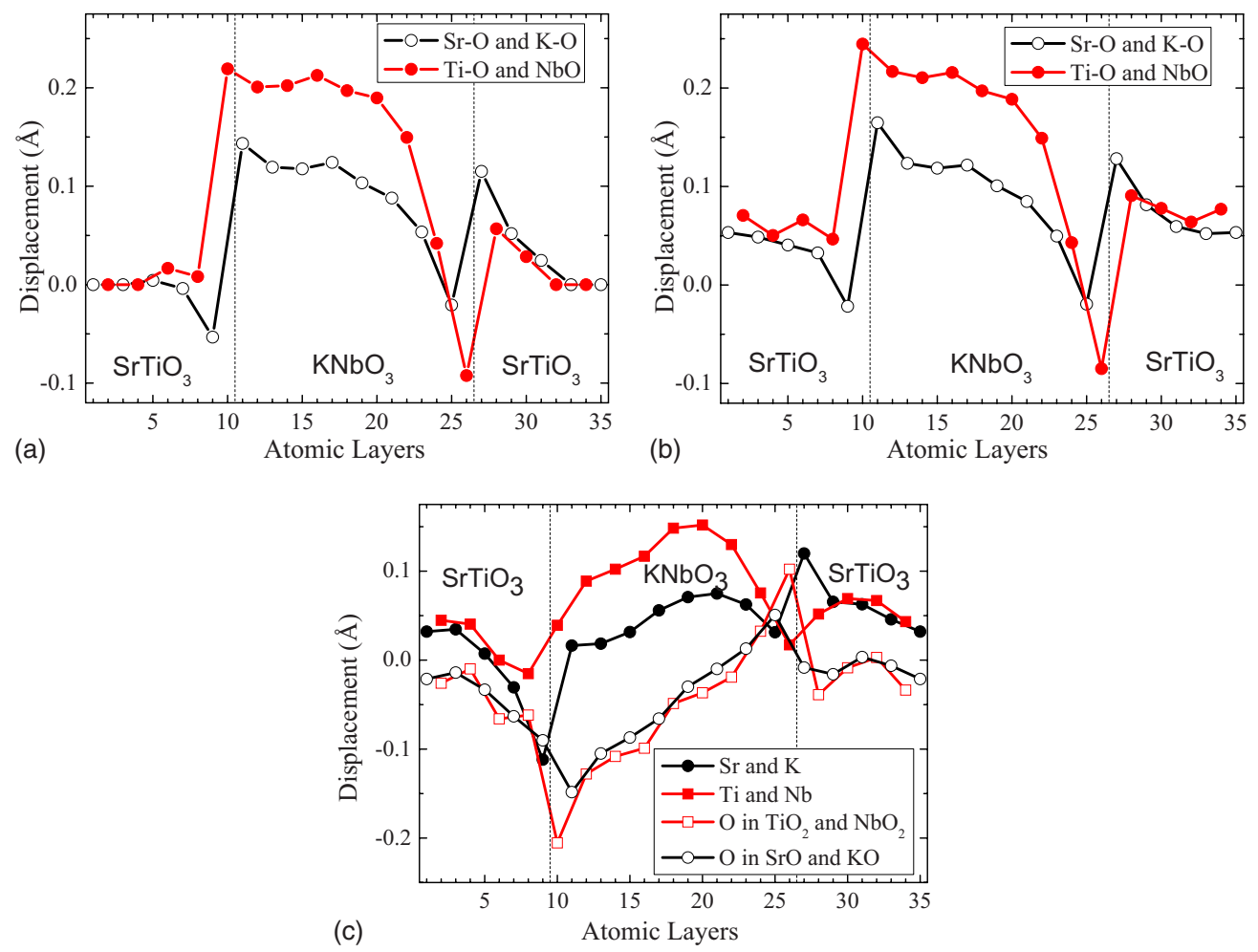

FIG. 4. (Color online) Cation $\left(\mathrm{Nb}, \mathrm{Ti}, \mathrm{K}\right.$, and $\mathrm{Sr}$ ) displacements with respect to oxygen anions in $\left(\mathrm{KNbO}_{3}\right)_{8.5} /\left(\mathrm{SrTiO}_{3}\right)_{8.5}$ superlattice with (a) eight middle monolayers of $\mathrm{SrTiO}_{3}$ fixed, (b) with all the ions fully relaxed, and (c) displacements of all the ions in fully relaxed ferroelectric state. Open and solid symbols in (a) and (b) indicate $\mathrm{Sr}-\mathrm{O}(\mathrm{K}-\mathrm{O})$ and $\mathrm{Ti}-\mathrm{O}_{2}\left(\mathrm{Nb}-\mathrm{O}_{2}\right)$ displacements, respectively. Solid circles and squares in (c) indicate $\mathrm{Sr}(\mathrm{K})$ and $\mathrm{Ti}(\mathrm{Nb})$ displacements, respectively. Open circles and squares in (c) indicate displacements of $\mathrm{O}$ atoms in $\mathrm{SrO}(\mathrm{KO})$ and $\mathrm{TiO}_{2}\left(\mathrm{NbO}_{2}\right)$ layers, respectively. The two dashed vertical lines indicate left and right $\mathrm{NbO}_{2} / \mathrm{SrO}$ interfaces.

Further insight as to how polarization switching influences the density of the 2DEG can be obtained from the number of free electrons accumulated at the two interfaces. We calculate local charges using Wigner-Seitz (WS) spheres that are used by VASP to project the wave functions onto spherical harmonics to calculate partial DOS. ${ }^{68}$ Table I shows the number of occupied $\mathrm{Nb} 4 d$ and Ti $3 d$ states integrated from conduction-band minimum up to the Fermi energy and added for all the atoms from the middle layer up to the left and right interface. In the paraelectric
$\mathrm{KNbO}_{3} / \mathrm{SrTiO}_{3}$ superlattice, free carriers on left and right interface are calculated to be 0.29 electrons each per unit-cell area, which are equal due to the symmetry. In the ferroelectric $\mathrm{KNbO}_{3} / \mathrm{SrTiO}_{3}$ superlattice with middle layer of $\mathrm{SrTiO}_{3}$ fixed, free carriers on right and left interface are obtained to be 0.53 electrons and 0.05 electrons per unit-cell area. Comparing with the paraelectric state, extra 0.24 electrons are accumulated at the right interface and 0.24 electrons are removed from the left interface. Evidently the induced screening charge has an opposite sign to the polarization charge,
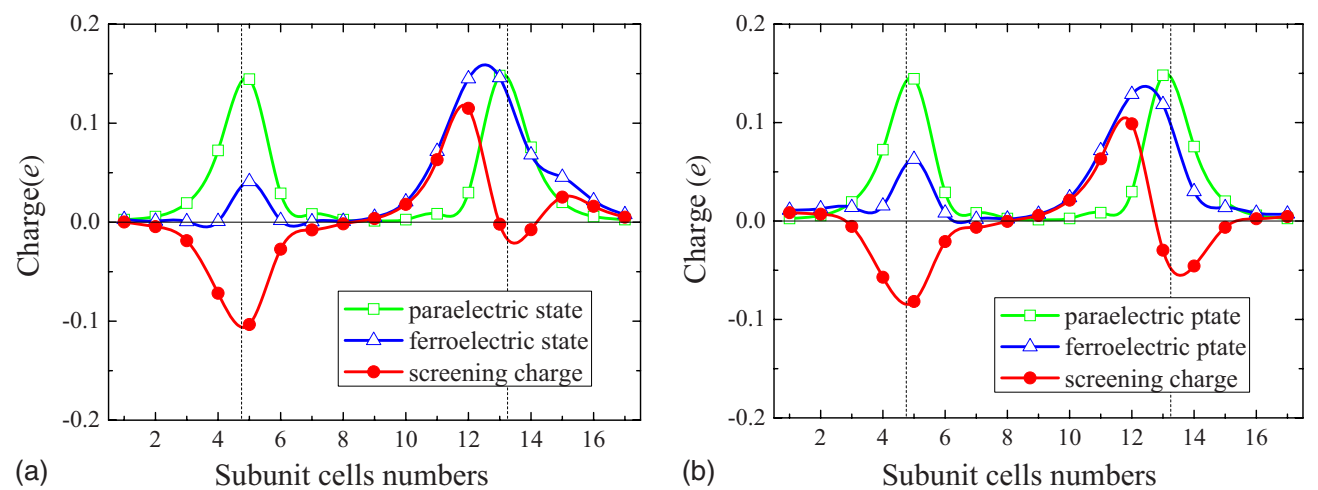

FIG. 5. (Color online) The free charge (in units of electron) on $\mathrm{Nb}$ and $\mathrm{Ti}$ atoms across the unit cell of $\left(\mathrm{KNbO}_{3}\right)_{8.5} /\left(\mathrm{SrTiO}_{3}\right)_{8.5}$ superlattices in paraelectric state and ferroelectric state when (a) the middle layers in $\mathrm{SrTiO}_{3}$ is fixed and (b) all the ions are fully relaxed. The screening charge is obtained by calculating the difference of the free charges on $\mathrm{Nb}$ and $\mathrm{Ti}$ atoms for ferroelectric and paraelectric states. The directions of polarizations for the ferroelectric state are from left to right. 
TABLE I. Number of electrons on $\mathrm{Nb}$ and $\mathrm{Ti}$ atoms per lateral unit-cell area at two interfaces in the $\mathrm{KNbO}_{3} / A \mathrm{TiO}{ }_{3}(A=\mathrm{Sr}$, $\mathrm{Ba}$, and $\mathrm{Pb})$ heterostructures without polarizations, with parallel and with antiparallel polarizations in each constituent.

\begin{tabular}{|c|c|c|c|c|c|c|c|c|c|c|}
\hline & & \multicolumn{3}{|c|}{ Ferroelectric: right interface } & \multicolumn{3}{|c|}{ Ferroelectric: left interface } & \multicolumn{3}{|c|}{ Paraelectric: either interface } \\
\hline \multicolumn{2}{|c|}{ Heterostructure } & $\mathrm{Nb}$ & $\mathrm{Ti}$ & Total & $\mathrm{Nb}$ & $\mathrm{Ti}$ & Total & $\mathrm{Nb}$ & $\mathrm{Ti}$ & Total \\
\hline \multirow[t]{4}{*}{ Parallel polarizations } & $\begin{array}{l}\mathrm{KNbO}_{3} / \mathrm{SrTiO}_{3}: \\
\text { full relaxation }\end{array}$ & 0.36 & 0.07 & 0.43 & 0.09 & 0.06 & 0.15 & 0.19 & 0.10 & 0.29 \\
\hline & $\begin{array}{c}\mathrm{KNbO}_{3} / \mathrm{SrTiO}_{3}: \\
\text { middle } \mathrm{SrTiO}_{3} \\
\text { layers fixed }\end{array}$ & 0.39 & 0.14 & 0.53 & 0.05 & 0.01 & 0.05 & 0.19 & 0.10 & 0.29 \\
\hline & $\mathrm{KNbO}_{3} / \mathrm{PbTiO}_{3}$ & 0.00 & 0.00 & 0.00 & 0.25 & 0.47 & 0.72 & 0.16 & 0.11 & 0.27 \\
\hline & $\mathrm{KNbO}_{3} / \mathrm{BaTiO}_{3}$ & 0.25 & 0.03 & 0.28 & 0.12 & 0.15 & 0.27 & 0.17 & 0.09 & 0.26 \\
\hline Antiparallel polarizations & $\mathrm{KNbO}_{3} / \mathrm{BaTiO}_{3}$ & 0.46 & 0.12 & 0.58 & 0.00 & 0.00 & 0.00 & 0.19 & 0.09 & 0.26 \\
\hline
\end{tabular}

thereby counteracting the depolarizing electric field. The polarization charge density $\sigma_{P}$ can be estimated from the polarization of $\mathrm{KNbO}_{3}$ in middle layer using the Berry phase method which gives $P_{\mathrm{KNbO} 3}=0.41 \mathrm{C} / \mathrm{m}^{2}$. This corresponds to the polarization charge per unit-cell area of $\sigma_{P}=0.39 e$. The WS charge density at the two interfaces changes by $0.24 e$ per unit-cell area in going from paraelectric to ferroelectric state. The WS charge density $(0.29 e$ per unit-cell area) underestimates the actual charge density $(0.5 e$ per unitcell area) in the paraelectric state. When scaled with the factor $0.5 / 0.29$, the change in charge at either interface becomes $0.41 e$ per unit-cell area which is about the same as the polarization charge density.

In the heterostructure with full relaxation, the difference of free charges between two interfaces is not as much as that when middle layer of $\mathrm{SrTiO}_{3}$ is fixed due to the small induced polarization in $\mathrm{SrTiO}_{3}$. We obtain the polarization of $\mathrm{SrTiO}_{3}$ from polar displacements [Fig. 4(b)], using the Berry-phase method, of about $0.17 \mathrm{C} / \mathrm{m}^{2}$. From the difference in the polarizations in $\mathrm{KNbO}_{3}$ and $\mathrm{SrTiO}_{3}$ of $0.24 \mathrm{C} / \mathrm{m}^{2}$, the polarization charge per unit-cell area is $\sigma_{P}$ $=0.23 e$, which is about the same as the screening charge density of $0.14 e$ per unit-cell area scaled with the factor $0.5 /$ 0.29 .

\section{B. $\mathrm{PbTiO}_{3} / \mathrm{KNbO}_{3}$}

Here we study electronic properties at the polar interfaces in the $\left(\mathrm{KNbO}_{3}\right)_{8.5} /\left(\mathrm{PbTiO}_{3}\right)_{8.5}$ heterostructure. When both subunits are in a paraelectric state, similar to the $\left(\mathrm{NbO}_{2}\right)^{+} /(\mathrm{SrO})^{0}$ interface in the $\mathrm{KNbO}_{3} / \mathrm{SrTiO}_{3}$ heterostructure, the presence of polar interfaces leads to the formation of 2DEG at the $\left(\mathrm{NbO}_{2}\right)^{+} /(\mathrm{PbO})^{0}$ interfaces in the $\mathrm{KNbO}_{3} / \mathrm{PbTiO}_{3}$ heterostructure. This is evident from Figs. 6(a) and 6(d), which show the Nb $4 d$ and Ti $3 d$ DOS indicating the largest local DOS at the Fermi energy at the monolayers located close to the interface $(l=1$ and $k=2)$. The local DOS decreases with the distance from the interface. The calculated free-carrier densities are 0.27 electrons per unit-cell area on both interfaces as shown in Table I.

Figure 7 shows the ferroelectric displacements of the cations $(\mathrm{Nb}, \mathrm{Ti}, \mathrm{K}$, and $\mathrm{Pb})$ relative to the oxygen anions in the heterostructure. It can be seen that in the
$\left(\mathrm{KNbO}_{3}\right)_{8.5} /\left(\mathrm{PbTiO}_{3}\right)_{8.5}$ heterostructure, the spontaneous polarization of strained $\mathrm{PbTiO}_{3}$ exceeds significantly the polarization of a $\mathrm{KNbO}_{3}$ subunit, which leads to positive and negative polarization charge on the left and right interfaces, respectively, even though the polarization for the system points to the right. As follows from our discussion of the $\mathrm{KNbO}_{3} / \mathrm{SrTiO}_{3}$ heterostructure, this leads to more free charges at the left interface and less free charges at the right interface to compensate the polarization charges.

It is seen from Figs. 6(b), 6(c), 6(e), and 6(f) that for $\left(\mathrm{KNbO}_{3}\right)_{8.5} /\left(\mathrm{PbTiO}_{3}\right)_{8.5}$ heterostructure, the DOS on both the $\mathrm{Nb}$ and $\mathrm{Ti}$ atoms at the Fermi energy is very large on the left interface [Figs. 6(c) and 6(f)] while that on the right interface is zero [Figs. 6(b) and 6(e)]. This indicates a ferroelectrically induced metal-insulator transition at the interface as a result of polarization reversal. The origin of this behavior can be explained by the large difference in polarizations on the two constituents of the heterostructure. The polarizations in the middle of $\mathrm{KNbO}_{3}$ and $\mathrm{PbTiO}_{3}$ in the superlattice are 0.47 and $1.09 \mathrm{C} / \mathrm{m}^{2}$, respectively. This result in a negative polarization charge of $0.58 e$ per unit-cell area is at the right interface which expels all the free carriers of $0.5 e$ per unit-cell area on that interface leading to an insulating right interface. This metal-insulator transition at the interface is visualized by the free charge distribution in Fig. 8. Thus a metal-insulator transition at the interface with polarization reversal is expected at the interface of the two constituents with a large difference in their polarizations.

\section{C. $\mathrm{BaTiO}_{3} / \mathrm{KNbO}_{3}$}

Similar to the polar interfaces considered above, the $\mathrm{KNbO}_{3} / \mathrm{BaTiO}_{3}$ system exhibits a 2DEG at the $\left(\mathrm{NbO}_{2}\right)^{+} /(\mathrm{BaO})^{0}$ interface. This is seen from the $\mathrm{Nb} 4 d$ and Ti $3 d$ DOS shown in Figs. 9(a) and 9(d) for the heterostructure in a paraelectric state. Again we see the largest occupied DOS near the Fermi energy at the interfacial layers which decreases when moving away from the interface. The calculated free-carrier densities are $0.26 e$ per unit-cell area on both interfaces as shown in Table I.

When ferroelectric state is developed in the $\mathrm{KNbO}_{3} / \mathrm{BaTiO}_{3}$ heterostructure the ferroelectric displacements in $\mathrm{KNbO}_{3}$ and $\mathrm{BaTiO}_{3}$ appeared to be similar in mag- 

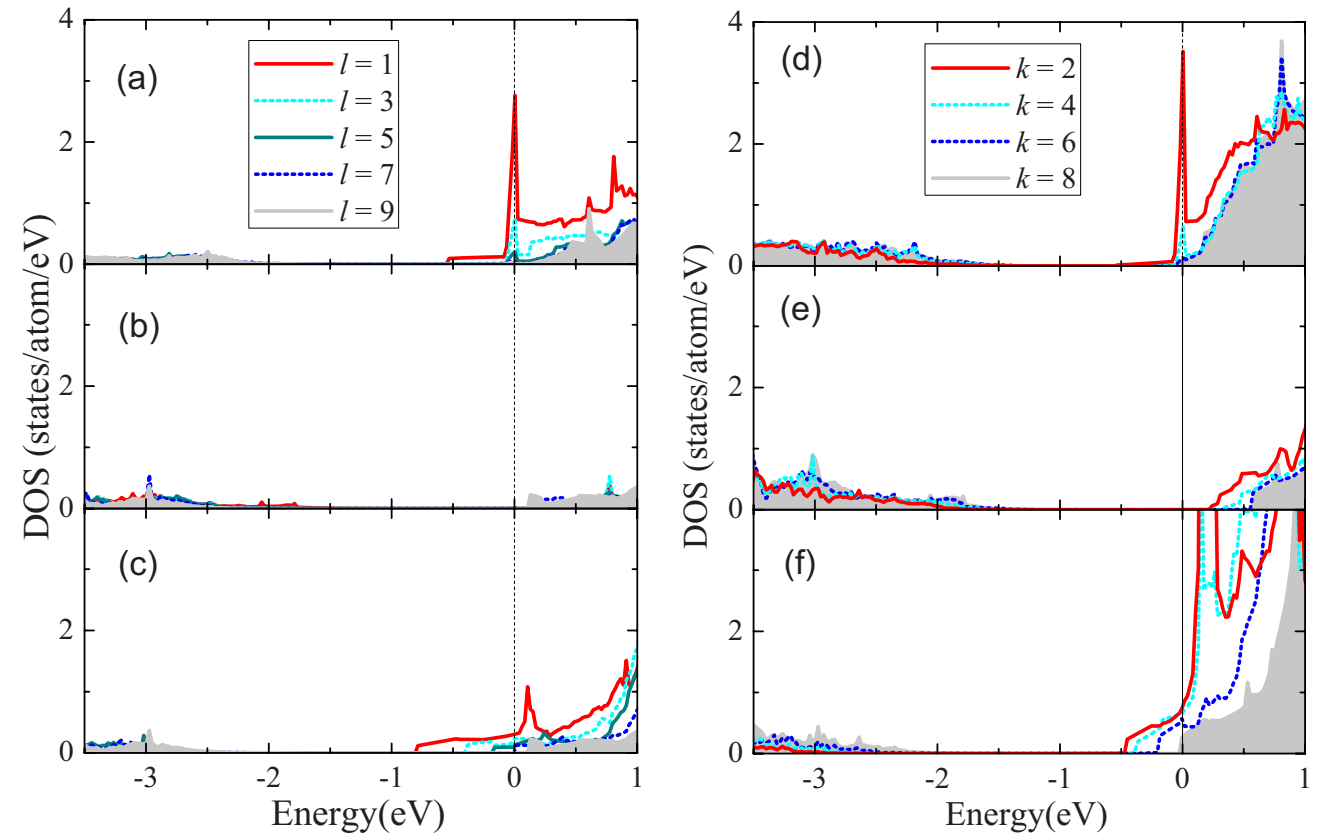

FIG. 6. (Color online) Layer-projected DOS on [(a)-(c)] $4 d$ orbitals of Nb atoms and [(d)-(f)] $3 d$ orbitals of Ti atoms located in different monolayers $l$ and $k$, respectively, from the $\left(\mathrm{NbO}_{2}\right)^{+} /(\mathrm{PbO})^{0}$ interface in the $[(\mathrm{a})$ and $(\mathrm{e})]\left(\mathrm{KNbO}_{3}\right)_{8.5} /\left(\mathrm{PbTiO}_{3}\right)_{8.5}$ superlattice for paraelectric state, [(b) and (e)] ferroelectric state and right interface, and [(c) and (f)] ferroelectric state and left interface. Layer number indicates the layer from the interface. The shaded plots are the DOS of atoms in the central monolayer. The zero along the horizontal axis refers to the Fermi energy

nitude. This is seen from Fig. 10(a) which shows the displacements of the cations $(\mathrm{Nb}, \mathrm{Ti}, \mathrm{K}$, and $\mathrm{Ba})$ relative to the oxygen anions. This leads to the comparable polarizations of strained $\mathrm{BaTiO}_{3}$ and $\mathrm{KNbO}_{3}$ in the heterostructure, mirroring the similarity of their bulk polarizations $(0.41$ and $0.43 \mathrm{C} / \mathrm{m}^{2}$ for $\mathrm{KNbO}_{3}$ and $\mathrm{BaTiO}_{3}$, respectively). This behavior is different from $\mathrm{KNbO}_{3} / \mathrm{SrTiO}_{3}$ and $\mathrm{KNbO}_{3} / \mathrm{PbTiO}_{3}$ systems, where the polarization discontinuity was pronounced at the interfaces.

The similar polarization values of $\mathrm{BaTiO}_{3}$ and $\mathrm{KNbO}_{3}$ lead to the net polarization charge of almost zero at the two interfaces for parallel polarizations resulting in the small difference in the density of free carriers at two interfaces as indicated in Table I. We note that the layer-resolved DOS of

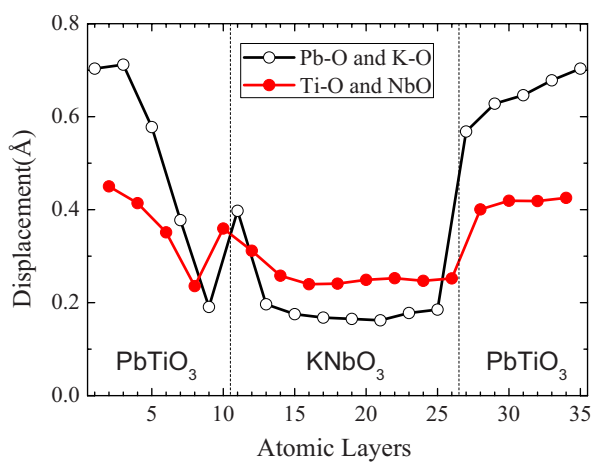

FIG. 7. (Color online) Cation ( $\mathrm{Nb}, \mathrm{Ti}, \mathrm{K}$, and $\mathrm{Pb}$ ) displacements with respect to oxygen anions in $\left(\mathrm{KNbO}_{3}\right)_{8.5} /\left(\mathrm{PbTiO}_{3}\right)_{8.5}$ superlattice. Open and solid and symbols indicate $\mathrm{Pb}-\mathrm{O}(\mathrm{K}-\mathrm{O})$ and $\mathrm{Ti}-\mathrm{O}_{2}$ $\left(\mathrm{Nb}-\mathrm{O}_{2}\right)$ displacements, respectively. The two dashed vertical lines indicate left and right $\left(\mathrm{NbO}_{2}\right)^{0} /(\mathrm{PbO})^{0}$ interfaces.
Ti is larger at the left interface than at the right interface while it is opposite for the layer-resolved DOS of $\mathrm{Nb}$ as is clearly seen from the comparison of Figs. 9(b) and 9(c) and Figs. 9(e) and 9(f). This asymmetric behavior is a direct result of opposite screening charges extending to several layers away from the interfaces to compensate the inhomogeneous polarization charge in each constituent. This can be seen from the screening charge distribution in each constituent in Fig. 11(a).

We see, therefore, that there is no pronounced switching effect at the conducting $\mathrm{NbO}_{2} / \mathrm{BaO}$ interface in the $\mathrm{KNbO}_{3} / \mathrm{BaTiO}_{3}$ heterostructure when polarizations of the two constituents are in the same direction. However, we pre-

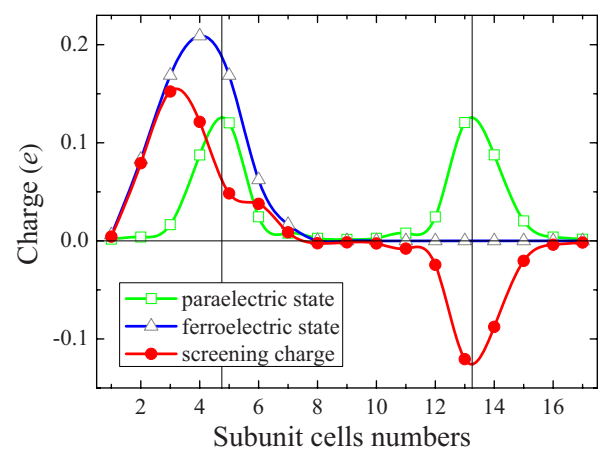

FIG. 8. (Color online) The free charge (in units of electron) on $\mathrm{Nb}$ and $\mathrm{Ti}$ atoms across the unit cell of $\left(\mathrm{KNbO}_{3}\right)_{8.5} /\left(\mathrm{PbTiO}_{3}\right)_{8.5}$ superlattices in paraelectric state and ferroelectric state. The screening charge is the difference of the free charges on $\mathrm{Nb}$ and $\mathrm{Ti}$ atoms for ferroelectric and paraelectric states. The direction of polarization for ferroelectric state is from left to right. 

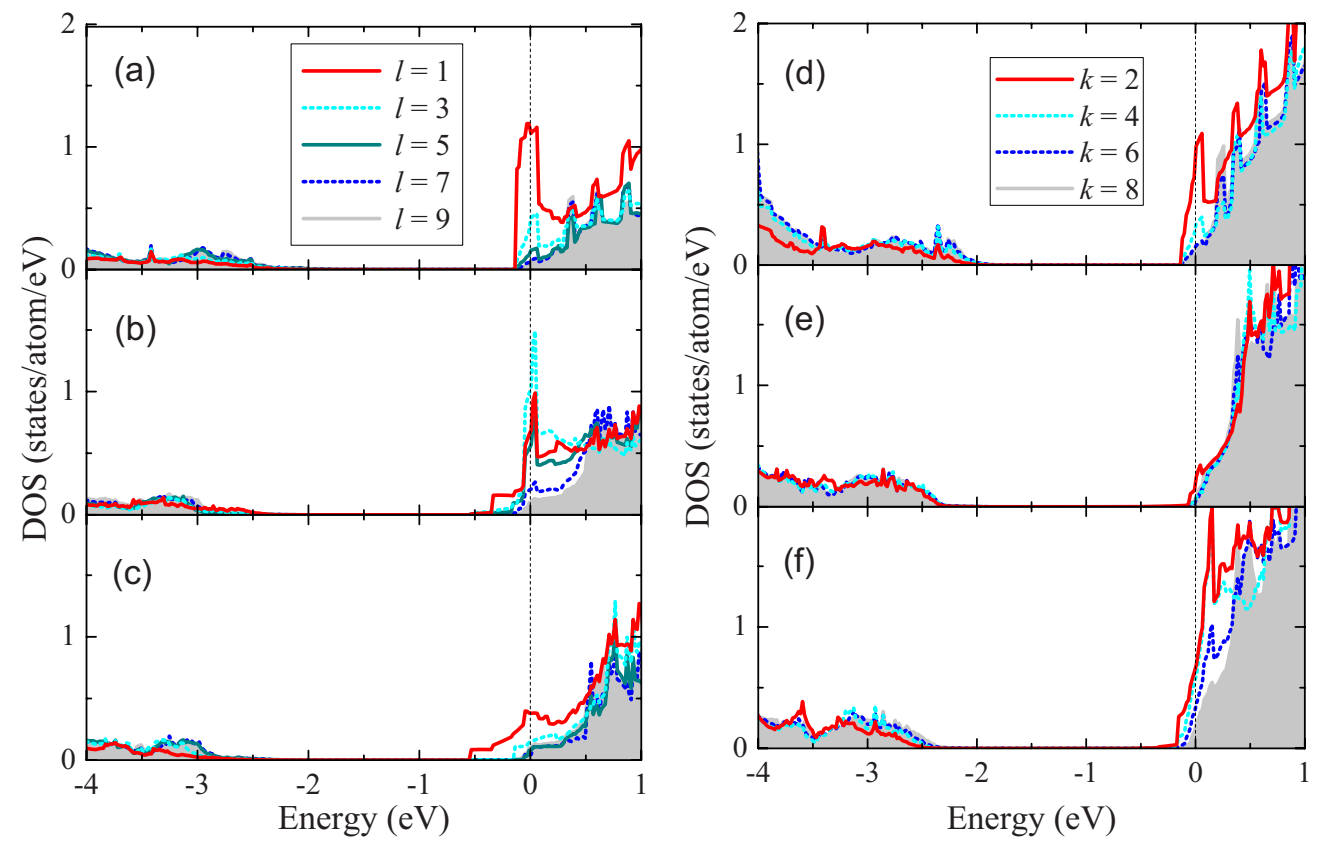

FIG. 9. (Color online) Layer-projected DOS on [(a)-(c)] $4 d$ orbitals of Nb atoms and [(d)-(f)] $3 d$ orbitals of Ti atoms located in different monolayers $l$ and $k$, respectively, away from the $\left(\mathrm{NbO}_{2}\right)^{+} /(\mathrm{BaO})^{0}$ interface in the $\left(\mathrm{KNbO}_{3}\right)_{8.5} /(\mathrm{BaTiO})_{8.5}$ superlattice for $[(\mathrm{a})$ and (e) paraelectric state, [(b) and (e)] ferroelectric state and right interface, and [(c) and (f)] ferroelectric state and left interface. Layer number indicates the layer from the interface. The shaded plots are the DOS of atoms in the central monolayer. The zero along the horizontal axis refers to the Fermi energy.

dict a metal-insulator transition at $\mathrm{NbO}_{2} / \mathrm{BaO}$ interface with polarizations reversal when polarizations in $\mathrm{KNbO}_{3}$ and $\mathrm{BaTiO}_{3}$ point opposite to each other. It has been predicted that while electrical-compatibility constraints normally prevent head-to-head and tail-to-tail domain walls from forming in ferroelectric materials, such domain walls could be stabilized by delta doping supplying a substitutional charge density that provides a cancellation of the bound polarization charge ${ }^{69}$ In our case the stabilization of the domains with the opposite polarization orientation is provided by the presence of the free charges at the interfaces. This antiparallel polarization state of $\mathrm{KNbO}_{3} / \mathrm{BaTiO}_{3}$ heterostructures is stable with respect to the paraelectric states by energies $-0.86 \mathrm{eV} /$ supercell. On the left interface, the polarizations

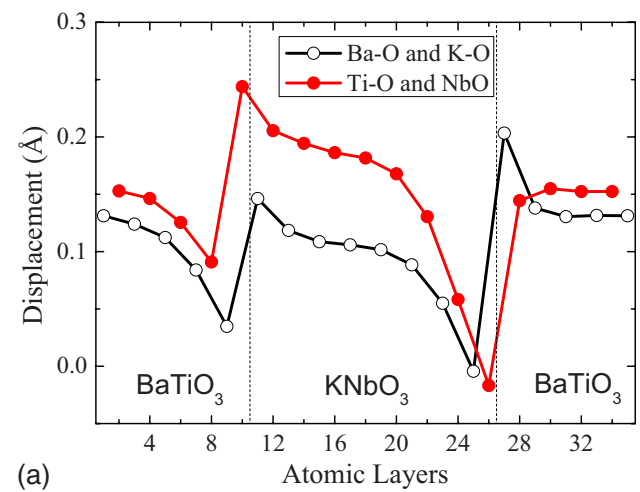

of $\mathrm{BaTiO}_{3}$ and $\mathrm{KNbO}_{3}$ are pointing away from the $\mathrm{NbO}_{2} / \mathrm{BaO}$ interface while on the right interface the polarizations are pointing toward interface as shown in Fig. 2(b). We find that the total energy of the superlattice in the parallel polarization state is lower by $0.32 \mathrm{eV} /$ supercell than that in the antiparallel state. The polarizations of $\mathrm{BaTiO}_{3}$ and $\mathrm{KNbO}_{3}$ in the heterostructure are -0.26 and $0.25 \mathrm{C} / \mathrm{m}^{2}$, respectively, causing $-0.49 e$ and $0.49 e$ polarization charges per unit-cell area on the left and right interface, respectively. The magnitude of the polarization charges is close to that of the free carriers (i.e., $0.5 e$ ) at the interfaces in paraelectric heterostructure. Thus, to stabilize the ferroelectric state with the antiparallel polarization almost all the free charge needs to be removed from one interface and placed to the other inter-

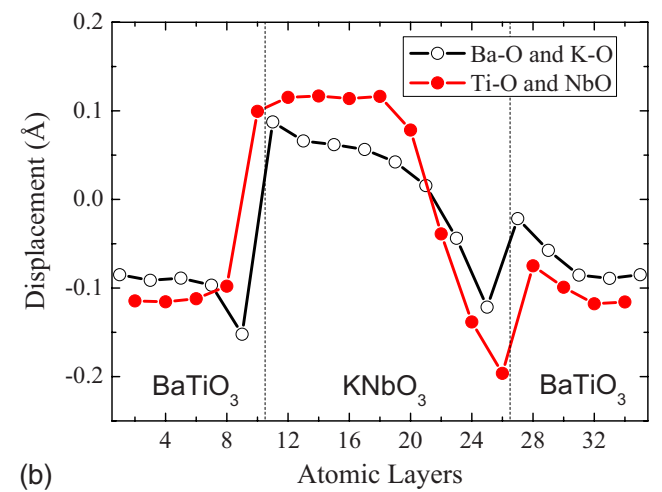

FIG. 10. (Color online) Cation $(\mathrm{Nb}, \mathrm{Ti}, \mathrm{K}$, and $\mathrm{Ba})$ displacements with respect to oxygen anions in $\left(\mathrm{KNbO}_{3}\right)_{8.5} /\left(\mathrm{BaTiO}_{3}\right)_{8.5}$ superlattice with (a) parallel polarizations pointing from left to right and with (b) antiparallel polarizations pointing toward each other. Open and solid symbols indicate $\mathrm{Ba}-\mathrm{O}(\mathrm{K}-\mathrm{O})$ and $\mathrm{Ti}_{-} \mathrm{O}_{2}\left(\mathrm{Nb}-\mathrm{O}_{2}\right)$ displacements, respectively. The two dashed vertical lines indicate left and right $\left(\mathrm{NbO}_{2}\right)^{0} /(\mathrm{BaO})^{0}$ interfaces. 

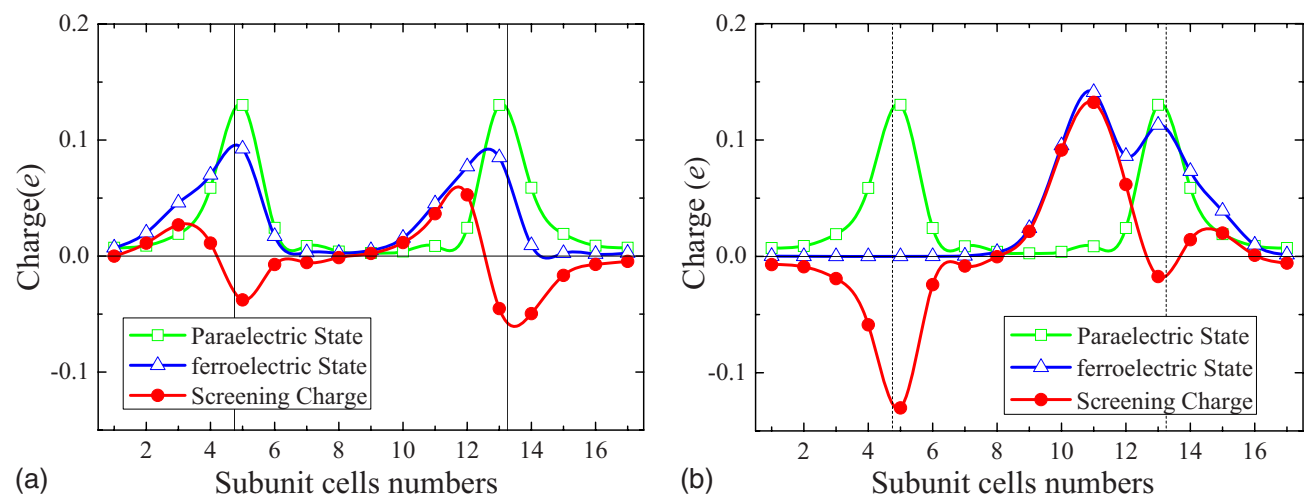

FIG. 11. (Color online) The free charge (in units of electron) on $\mathrm{Nb}$ and $\mathrm{Ti}$ atoms across the unit cell of $(\mathrm{KNbO} 3)_{8.5} /(\mathrm{BaTiO} 3)_{8.5}$ superlattices in paraelectric state and ferroelectric state with (a) polarizations in the same direction from left to right and (b) opposite polarizations. The screening charge is the difference of the free charges on $\mathrm{Nb}$ and $\mathrm{Ti}$ atoms for ferroelectric and paraelectric states.

face. This causes a metal-insulator transition at the interface by the polarization reversal as explained in the section describing a $\mathrm{KNbO}_{3} / \mathrm{PbTiO}_{3}$ heterostructure.

The presence of the ferroelectrically induced metalinsulator transition at the interface is seen from the calculated charge distribution in the $\mathrm{KNbO}_{3} / \mathrm{BaTiO}_{3}$ heterostructure with antiparallel polarization as shown in Fig. 11(b). It is seen that while in the paraelectric state the free charge is equally localized at the two interfaces, the antiparallel ferroelectric polarization state eliminates the charge from the left interface and places it to the right interface. It is notable that the distribution of this charge is not symmetric with respect to the interface reflecting complex structural relaxations at this interface evident from Fig. 10(b).

\section{SUMMARY}

We have explored the formation of 2DEG and its dependence on the electric polarization at the $\left(\mathrm{NbO}_{2}\right)^{+} /(A \mathrm{O})^{0}$ interface in the $\left(\mathrm{KNbO}_{3}\right) /\left(A \mathrm{TiO}_{3}\right)(A=\mathrm{Sr}, \mathrm{Pb}$, and $\mathrm{Ba})$ oxide heterostructures using first-principles methods. The chosen structures have ferroelectric constituents besides having polar discontinuity similar to that at the interface in $\left(\mathrm{LaAlO}_{3}\right) /\left(\mathrm{SrTiO}_{3}\right)$ heterostructure. The interfaces are found to have occupied $\mathrm{Nb} 4 d$ states and Ti $3 d$ states around the Fermi energy leading to conducting interfaces with $n$-type carriers.

We have predicted that the conducting properties at the two interfaces in these systems are influenced by ferroelec- tricity. Switching the ferroelectric polarization orientation may cause significant changes in the carrier density and consequently the conductivity of the interfacial 2DEG. The effect of the polarization reversal on the interface carrier density is understood in terms of screening by $2 \mathrm{DEG}$. The magnitude of the effect is controlled by contrast between polarizations of the two constituents of the heterostructure: the larger is the difference in the two polarizations, the bigger is the effect. Our calculations predict that a heterostructure with ferroelectric constituents can be designed such that the density of the 2DEG at the interface can be controlled by an external electric field including the possibility of switchable metal-insulator transition at the interface. These effects may be useful in controlling the interfacial conducting properties in ferroelectric oxide heterostructures by external electric fields.

\section{ACKNOWLEDGMENTS}

The authors thank Chang-Beom Eom and Mark Rzchowski for helpful discussions. This work was supported by National Science Foundation (Grant No. DMR-0906443), the Nanoelectronics Research Initiative through the Materials Research Science and Engineering Center at the University of Nebraska (NSF-DMR under Grant No. 0820521), and the Nebraska Research Initiative. Computations were performed utilizing the Research Computing Facility of the University of Nebraska-Lincoln.
${ }^{1}$ Y. Tokura and H. Y. Hwang, Nature Mater. 7, 694 (2008).

${ }^{2}$ J. W. Reiner, F. J. Walker, and C. H. Ahn, Science 323, 1018 (2009).

${ }^{3}$ A. Ohtomo and H. Y. Hwang, Nature (London) 427, 423 (2004).

${ }^{4}$ M. Basletic, J.-L. Maurice, C. Carrétéro, G. Herranz, O. Copie, M. Bibes, E. Jacquet, K. Bouzehouane, S. Fusil, and A. Barthélémy, Nature Mater. 7, 621 (2008).

${ }^{5}$ S. Thiel, G. Hammerl, A. Schmehl, C. W. Schneider, and J. Mannhart, Science 313, 1942 (2006).

${ }^{6}$ C. Cen, S. Thiel, J. Mannhart, and J. Levy, Science 323, 1026 (2009).
${ }^{7}$ A. Brinkman, M. Huijben, M. van Zalk, J. Huijben, U. Zeitler, J. C. Maan, W. G. van der Wiel, G. Rijnders, D. H. A. Blank, and H. Hilgenkamp, Nature Mater. 6, 493 (2007).

${ }^{8}$ N. Reyren, S. Thiel, A. D. Caviglia, L. F. Kourkoutis, G. Hammerl, C. Richter, C. W. Schneider, T. Kopp, A. S. Ruetschi, D. Jaccard, M. Gabay, D. A. Muller, J. M. Triscone, and J. Mannhart, Science 317, 1196 (2007).

${ }^{9}$ Y. Hotta, T. Susaki, and H. Y. Hwang, Phys. Rev. Lett. 99, 236805 (2007).

${ }^{10}$ A. Kalabukhov, R. Gunnarsson, J. Börjesson, E. Olsson, T. Claeson, and D. Winkler, Phys. Rev. B 75, 121404(R) (2007). 
${ }^{11}$ W. Siemons, G. Koster, H. Yamamoto, W. A. Harrison, G. Lucovsky, T. H. Geballe, D. H. A. Blank, and M. R. Beasley, Phys. Rev. Lett. 98, 196802 (2007).

${ }^{12}$ G. Herranz, M. Basletić, M. Bibes, C. Carrétéro, E. Tafra, E. Jacquet, K. Bouzehouane, C. Deranlot, A. Hamzić, J.-M. Broto, A. Barthélémy, and A. Fert, Phys. Rev. Lett. 98, 216803 (2007).

${ }^{13} \mathrm{~K}$. Yoshimatsu, R. Yasuhara, H. Kumigashira, and M. Oshima, Phys. Rev. Lett. 101, 026802 (2008).

${ }^{14}$ O. Copie, V. Garcia, C. Bödefeld, C. Carrétéro, M. Bibes, G. Herranz, E. Jacquet, J.-L. Maurice, B. Vinter, S. Fusil, K. Bouzehouane, H. Jaffrès, and A. Barthélémy, Phys. Rev. Lett. 102, 216804 (2009).

${ }^{15}$ C. Bell, S. Harashima, Y. Hikita, and H. Y. Hwang, Appl. Phys. Lett. 94, 222111 (2009).

${ }^{16}$ Z. S. Popovic and S. Satpathy, Phys. Rev. Lett. 94, 176805 (2005); Z. S. Popovic, S. Satpathy, and R. M. Martin, ibid. 101, 256801 (2008); B. R. K. Nanda and S. Satpathy, ibid. 101, 127201 (2008).

${ }^{17}$ M. S. Park, S. H. Rhim, and A. J. Freeman, Phys. Rev. B 74, 205416 (2006).

${ }^{18}$ S. Okamoto, A. J. Millis, and N. A. Spaldin, Phys. Rev. Lett. 97, 056802 (2006).

${ }^{19}$ J. M. Albina, M. Mrovec, B. Meyer, and C. Elsasser, Phys. Rev. B 76, 165103 (2007).

${ }^{20}$ R. Pentcheva and W. E. Pickett, Phys. Rev. B 74, 035112 (2006); Phys. Rev. Lett. 99, 016802 (2007); 102, 107602 (2009).

${ }^{21}$ K. Janicka, J. P. Velev, and E. Y. Tsymbal, J. Appl. Phys. 103, 07 B508 (2008); Phys. Rev. Lett. 102, 106803 (2009).

${ }^{22}$ J. Lee and A. A. Demkov, Phys. Rev. B 78, 193104 (2008).

${ }^{23}$ S. Ishibashi and K. Terakura, J. Phys. Soc. Jpn. 77, 104706 (2008).

${ }^{24}$ Z. Zhong and P. J. Kelly, EPL 84, 27001 (2008).

${ }^{25}$ Y. Wang, M. K. Niranjan, J. D. Burton, J. M. An, K. D. Belashchenko, and E. Y. Tsymbal, Phys. Rev. B 79, 212408 (2009).

${ }^{26}$ H. Chen, A. M. Kolpak, and S. Ismail-Beigi, Phys. Rev. B 79, 161402(R) (2009).

${ }^{27}$ N. C. Bristowe, E. Artacho, and P. B. Littlewood, Phys. Rev. B 80, 045425 (2009).

${ }^{28}$ N. Nakagawa, H. Y. Hwang, and D. A. Muller, Nature Mater. 5, 204 (2006).

${ }^{29}$ G. A. Baraff, J. A. Appelbaum, and D. R. Hamann, Phys. Rev. Lett. 38, 237 (1977).

${ }^{30}$ W. A. Harrison, E. A. Kraut, J. R. Waldrop, and R. W. Grant, Phys. Rev. B 18, 4402 (1978).

${ }^{31}$ T. Higuchi, Y. Hotta, T. Susaki, A. Fujimori, and H. Y. Hwang, Phys. Rev. B 79, 075415 (2009).

${ }^{32}$ M. Takizawa, Y. Hotta, T. Susaki, Y. Ishida, H. Wadati, Y. Takata, K. Horiba, M. Matsunami, S. Shin, M. Yabashi, K. Tamasaku, Y. Nishino, T. Ishikawa, A. Fujimori, and H. Y. Hwang, Phys. Rev. Lett. 102, 236401 (2009).

${ }^{33}$ A. Tsukazaki, A. Ohtomo, T. Kita, Y. Ohno, H. Ohno, and M. Kawasaki, Science 315, 1388 (2007).

${ }^{34}$ T. Kamiya and M. Kawasaki, MRS Bull. 33, 1061 (2008).

${ }^{35}$ M. K. Niranjan, Y. Wang, S. S. Jaswal, and E. Y. Tsymbal, Phys. Rev. Lett. 103, 016804 (2009).

${ }^{36}$ C.-G. Duan, S. S. Jaswal, and E. Y. Tsymbal, Phys. Rev. Lett. 97, 047201 (2006).

${ }^{37}$ C.-G. Duan, J. P. Velev, R. F. Sabirianov, W. N. Mei, S. S. Jaswal, and E. Y. Tsymbal, Appl. Phys. Lett. 92, 122905 (2008).
${ }^{38}$ M. K. Niranjan, J. P. Velev, C.-G. Duan, S. S. Jaswal, and E. Y. Tsymbal, Phys. Rev. B 78, 104405 (2008); M. K. Niranjan, J. D. Burton, J. P. Velev, S. S. Jaswal, and E. Y. Tsymbal, Appl. Phys. Lett. 95, 052501 (2009).

${ }^{39}$ M. Y. Zhuravlev, R. F. Sabirianov, S. S. Jaswal, and E. Y. Tsymbal, Phys. Rev. Lett. 94, 246802 (2005); 102, 169901 (2009); M. Y. Zhuravlev, S. S. Jaswal, E. Y. Tsymbal, and R. F. Sabirianov, Appl. Phys. Lett. 87, 222114 (2005).

${ }^{40}$ E. Y. Tsymbal and H. Kohlstedt, Science 313, 181 (2006).

${ }^{41}$ J. P. Velev, C.-G. Duan, K. D. Belashchenko, S. S. Jaswal, and E. Y. Tsymbal, Phys. Rev. Lett. 98, 137201 (2007).

${ }^{42}$ J. P. Velev, C.-G. Duan, J. D. Burton, A. Smogunov, M. K. Niranjan, E. Tosatti, S. S. Jaswal, and E. Y. Tsymbal, Nano Lett. 9, 427 (2009).

${ }^{43}$ É. D. Murray and D. Vanderbilt, Phys. Rev. B 79, 100102(R) (2009).

${ }^{44}$ P. Hohenberg and W. Kohn, Phys. Rev. 136, B864 (1964).

${ }^{45}$ W. Kohn and L. J. Sham, Phys. Rev. 140, A1133 (1965).

${ }^{46}$ M. C. Payne, M. P. Teter, D. C. Allan, T. A. Arias, and J. D. Joannopoulos, Rev. Mod. Phys. 64, 1045 (1992).

${ }^{47}$ G. Kresse and J. Hafner, Phys. Rev. B 47, 558 (1993); 49, 14251 (1994).

${ }^{48}$ G. Kresse and J. Furthmüller, Comput. Mater. Sci. 6, 15 (1996); Phys. Rev. B 54, 11169 (1996).

${ }^{49}$ P. E. Blöchl, Phys. Rev. B 50, 17953 (1994).

${ }^{50}$ J. P. Perdew, K. Burke, and M. Ernzerhof, Phys. Rev. Lett. 78, 1396 (1997).

${ }^{51}$ H. J. Monkhorst and J. D. Pack, Phys. Rev. B 13, 5188 (1976).

${ }^{52}$ K. Nassau and A. E. Miller, J. Cryst. Growth 91, 373 (1988).

${ }^{53}$ C.-G. Duan, R. Sabirianov, W.-N. Mei, S. S. Jaswal, and E. Y. Tsymbal, Nano Lett. 6, 483 (2006).

${ }^{54}$ N. Sai, A. M. Kolpak, and A. M. Rappe, Phys. Rev. B 72, 020101(R) (2005).

${ }^{55}$ Y. Kuroiwa, S. Aoyagi, A. Sawada, J. Harada, E. Nishibori, M. Takata, and M. Sakata, Phys. Rev. Lett. 87, 217601 (2001).

${ }^{56}$ Y. Umeno, B. Meyer, C. Elsässer, and P. Gumbsch, Phys. Rev. B 74, 060101(R) (2006).

${ }^{57}$ R. D. King-Smith and D. Vanderbilt, Phys. Rev. B 47, 1651 (1993).

${ }^{58}$ O. Nakagawara, T. Shimuta, T. Makino, S. Arai, H. Tabata, and T. Kawai, Vacuum 66, 397 (2002).

${ }^{59}$ W. Kleemann, F. J. Schafer, and M. D. Fontana, Phys. Rev. B 30, 1148 (1984) and references therein.

${ }^{60}$ T. Morita and Y. Cho, Jpn. J. Appl. Phys. 43, 6535 (2004).

${ }^{61}$ J. B. Neaton and K. M. Rabe, Appl. Phys. Lett. 82, 1586 (2003).

${ }^{62}$ T. Neumann, G. Borstel, C. Scharfschwerdt, and M. Neumann, Phys. Rev. B 46, 10623 (1992).

${ }^{63}$ J. Robertson and C. W. Chen, Appl. Phys. Lett. 74, 1168 (1999).

${ }^{64}$ P. W. Peacock and J. Robertson, J. Appl. Phys. 92, 4712 (2002).

${ }^{65}$ R. E. Cohen, Nature (London) 362, 213 (1993).

${ }^{66}$ J. Junquera and Ph. Ghosez, Nature (London) 422, 506 (2003).

${ }^{67}$ J. H. Haeni, P. Irvin, W. Chang, R. Uecker, P. Reiche, Y. L. Li, S. Choudhury, W. Tian, M. E. Hawley, B. Craigo, A. K. Tagantsev, X. Q. Pan, S. K. Streiffer, L. Q. Chen, S. W. Kirchoefer, J. Levy, and D. G. Schlom, Nature (London) 430, 758 (2004).

${ }^{68}$ For WS radii we use the default values of $2.138,1.588,1.979$, $1.725,1.323,1.270$, and $0.820 \AA$ for the radii of $\mathrm{Sr}, \mathrm{K}, \mathrm{Ba}, \mathrm{Pb}$, $\mathrm{Ti}, \mathrm{Nb}$, and $\mathrm{O}$ atoms, respectively, provided by the input potential file in the VASP program.

${ }^{69}$ X. Wu and D. Vanderbilt, Phys. Rev. B 73, 020103(R) (2006). 